\title{
Quaternary DS-SSMA Communications Employing Generalized Detector over Rayleigh Fading Channels
}

\author{
VYACHESLAV TUZLUKOV \\ Department of Technical Maintenance of Aviation and Radio Electronic Equipment \\ Belarusian State Aviation Academy \\ 77, Uborevicha Str., 220096 Minsk \\ BELARUS \\ slava.tuzlukov@mail.ruｈttp://www.bgaa.by
}

\begin{abstract}
This paper deals with an employment of the complex modulators and generalized receivers constructed based on the generalized approach to signal processing (GASP) in noise in quaternary direct-sequence spread-spectrum multiple-access (DS-SSMA) communication systems with complex signature sequences in flat Rayleigh fading. Owing to availability of large sets of complex spreading sequences possessing perfect correlation characteristics, the interest to use the complex spreading sequences in DS-SSMA communication systems has increased. The complex spreading sequences investigated in the present paper are based on the recently introduced orthogonal unified complex Hadamard transform (UCHN) sequences. In the present paper, the compl- ex processing in modulators and generalized receivers is also employed with the purpose to take advantage of the correlation properties of complex signature sequences. We derive the average bit error rate (BER) for quarternary synchronous communications system based on the generalized approach to signal processing in noise first, and then the BER for quaternary asynchronous ones is evaluated using the characteristic function appro- ach. We presented some results based on the Gaussian approximation method for asynchronous communication systems constructed on the basis of the generalized approach to signal processing in noise Computer modeling results demonstrate that the communication systems using UCHT spreading sequences perform generally better
\end{abstract}

than the GO.LD sequences and the 4-phase family of sequences. Comparative analysis between the asynchronous communication systems constructed on the basis of the generalized approach to signal processing in noise demonstrates superiority over the asynchronous communication systems employing the correlation receiver.

Key-Words: - Generalized receiver, quaternary direct-sequence spread-spectrum multiple-access (DS-SSMA) communications, Rayleigh fading, unified complex Hadamard Transform (UCHN) complex sequences.

Received: October 31, 2019. Revised: March 12, 2020. Accepted: March 27, 2020. Published: March 31, 2020.

\section{Introduction}

In last several decades, the direct-sequence spreadspectrum multiple-access (DS-SSMA) technique has attracted a lot of attention as a transmission method providing spectrum efficiency, high system capacity, multipath propagation, interference robustness, and improved quality of service [1]-[3]. In DSSSMA communication systems, the characteristics of the spreading codes provide a crucial effect on the performance of the whole communication system [4], [5]. How much interference from other users is received at a receiver is determined by the signature sequences. Additionally, the signature sequences influence on the extraction capability of desired signal from noise-like spectrum. The orthogonal spreading sequences are characterized by the zero cross correlation for zero delay. This fact has attracted a great attention in research and applications.

The polyphase complex sequences such as Frank -Zadoff-Chu sequences in [2], [6], and [7] have the excellent correlation properties and can be $3 \mathrm{~dB}$ better than the binary real Gold sequences in the maxi- mum periodic correlation parameters. Also, the larger sets of complex sequences are available. Owing to the fact that the complex sequences exhibit the better autocorrelation and improved cross correlation properties than binary real sequences, the application of the complex sequences in DS-SSMA has a great research interest [8]-[10]. However, these complex sequences are non-orthogonal. They can be categorized as complex-valued pseudo-random spreading sequences. A set of the orthogonal 4-phase complex sequences that are derived from the unified complex Hadamard Transform (UCHT) matrix [11] was introduced in [10].

Therein, the correlation properties of the UCHT complex sequences were obtained, and simulation results were given by applying the UCHT sequences to the binary phase shift keying (BPSK) DS-SSMA systems over the additive white Gaussian noise (AWGN) channels. DS-SSMA systems and their performance evaluation techniques have been discussed in [1], [4] and [12]. In [8] and [9] the performance bounds for DS-SSMA systems with complex sig- 
nature sequences were investigated for the binary PSK data signalling and for $M$-ary PSK data signalling. The modulators and receivers discussed in these papers use the real processing. For instance, in the IS-95 CDMA mobile cellular system [1], both forward and reverse links use the forms of quadrature phase shift keying (QPSK) spread-spectrum modulation in which the same baseband binary signalling data stream modulates both in-phase (I) and quadrature $(\mathrm{Q})$ binary real spreading sequences, and the receiver may employ separate I and Q real processing of the spread-spectrum signal with binary real spreading sequences.

This paper adopts a new receiver based on the generalized approach to signal processing in noise [13]-[18] employing the complex processing instead of real. Hence, for the quaternary DS-SSMA systems, the modulators and generalized receivers are complex and not performed in separate in-phase and quadrature branches. All research results in the literature mentioned above are established over AWGN channels. It is well known that the fading and multiple-access interference (MAI) are two major sources of degradation in wireless and CDMA communication systems. Therefore, analysis of the DS-SSMA communication systems performance on fading channels is of considerable theoretical and practical interest.

Many papers deal with the error probability performance evaluations for binary DS-SSMA systems operating in fading channels with real binary spreading sequences [19]-[23]. In [19] the signal-to-noise ratio (SNR) is studied at the output of the correlation receiver for Rician fading channels. The performances of DS-SSMA systems over Rayleigh fading channels are investigated in [20] for deterministic binary sequences and in [22] for random binary sequences. The influence of Rician factor of $\kappa-\mu$ shortterm fading, $\kappa-\mu$ short-term fading severity parameter, Gamma long-term fading severity parameter and Gamma long-term fading correlation coefficient on level crossing rate is studied and discussed in [23] under consideration of the macrodiversity with selection combining receiver and two microdiversity maximum ratio combining receivers. However, there is no the result for the error probability performance analysis of quaternary DS-SSMA systems that operate in Rayleigh fading channels and employ the complex spreading sequence, as well as complex processing at the transmitters and receivers.

We carry out the comparative analysis between performances of the DS-SSMA communication systems based on the correlation and generalized receivers for this case. The main goal of this paper is to investigate the performance of the quaternary DS-
SSMA communication system based on the generalized approach to signal processing in noise with complex signature sequences in the presence of the flat Rayleigh fading employing the generalized receiver and compare the performance of the quaternary DSSSMA communication system employing the correlation receiver. The complex spreading sequences include the orthogonal quaternary UCHT complex sequences [11]. In this paper the bit error rate (BER) performance evaluation is first developed for the synchronous complex quaternary DS-SSMA systems employing the generalized receiver. Then the BER is evaluated for the asynchronous complex quarternary DS-SSMA systems employing the generalized receiver based on both the characteristic function approach and Gaussian approximation method.

The reminder of this paper is organized as follows. Section II presents some basic definitions and review of UCHT sequences. Section III studies the quaternary DS-SSMA system model with complex signature sequences in Rayleigh fading based on the generalized approach to signal processing in noise. The BER performance is evaluated in Section IV for quaternary synchronous and asynchronous systems with the complex signature sequences employing the generalized receiver under complex processing. Numerical results and comparative analysis of performance between the quaternary DS-SSMA communication system based on the generalized approach to signal processing in noise with complex signature sequences in the presence of the flat Rayleigh fading employing the generalized receiver and the quarternary DS-SSMA communication system employing the correlation receiver is presented in Section V. Some conclusion remarks are made in Section VI.

\section{Basic Definitions}

In this section, we will define the unified complex Hadamard transform (UCHT) matrix that generates orthogonal complex sequences by its rows. The UCHT matrix $U_{n}$ of order $N=2^{n}$ is the square matrix and can be constructed by the following form [9], [10]

$$
U_{n}=U_{1} \otimes U_{n-1}=\underbrace{U_{1} \otimes \cdots \otimes U_{1}}_{n \text { times }}
$$

where $\otimes$ denotes the Kronecker product of matrices; $U_{1}$ is defined as

$$
U_{1}=\left[\begin{array}{cc}
\mu_{1} & \mu_{1} \mu_{3} \\
\mu_{2} & -\mu_{2} \mu_{3}
\end{array}\right]
$$

with $\mu_{1}, \mu_{2}, \mu_{3} \in\{1,-1, j,-j\}$ and $j=\sqrt{-1}, \mu_{1}$. Note that $U_{1}$ satisfies to the condition

$$
U_{1} U_{1}^{*}=U_{1}^{*} U_{1}=2 I_{2}
$$


and

$$
\left|\operatorname{det} U_{1}\right|^{2}=2^{2},
$$

where $U_{1}^{*}$ represents the complex conjugate transpose of the matrix $U_{1}$. Hence, $U_{1}$ is the complex orthogonal Hadamard matrix and the UCHT matrix is complex orthogonal [24]. Moreover, it is obvious that $U_{1}$ is a unified form of 64 different matrices with elements in $\{ \pm 1, \pm j\}$, and among them, there are 8 matrices with all four different element values. UCHT matrices contain the Walsh-Hadamard transform (WHT) matrix as a special case with $\mu_{1}=1, \mu_{2}=1, \mu_{3}$ $=1$ in the matrix $U_{1}$.

In addition, since the elements of the matrix $U_{1}$ are confined to four values $\{ \pm 1, \pm j\}$, UCHT represents the mapping of four-valued integers into a unit circle of complex plane. For example, UCHT can be used as four-valued complex transform that maps four integers $(0,1,2,3)$ into $(1, j,-1,-j)[10]$. From the above discussion, 64 sets of orthogonal sequences can be generated depending on the various combinations of $\mu_{1}, \mu_{2}$ and $\mu_{3}$. Depending on whether $\mu_{3}$ is imaginary or real, these UCHT matrices can be categorized into two groups [11], i.e., UCHT with halfspectrum-property (HSP-UCHT) and UCHT without half-spectrum-property (NHSP-UCHT). Each group has 32 sets of UCHT matrices.

Let each row of one UCHT matrix be a complex sequence. Then the sequence $a^{(i)}$ represents the $i$-th row of the corresponding UCHT matrix, and the $m$ th element of $a^{(i)}$ is $a_{m}^{(i)}=u(i, m)$, where $u(i, m)$ is the element of the UCHT matrix. By repeating the UCHT sequences, $N=2^{n}$ orthogonal UCHT sequences with the periods of $N$ are produced. For any two periodic sequences $a^{(k)}$ and $a^{(i)}$ with period $N$, the aperiodic cross correlation function is defined in $[25]$ as

$$
R_{r, i}(l)= \begin{cases}\sum_{m=0}^{N-1-l} a_{m}^{(k)} a_{m+l}^{(i)}, & 0 \leq l \leq N-1 \\ \sum_{m=0}^{N-1+l} a_{m-l}^{(k)} a_{m}^{(i)^{\bullet}} & , 1-N \leq l<0 \\ 0, & |l| \geq N .\end{cases}
$$

If $k=i$, the cross correlation function is the autocorrelation function $R_{i}(l)=R_{i, i}(l)$.

Introduce some notation for the complex-valued random variables. The cumulative distribution function of a complex variable $Z$ is specified by giving the joint distribution of its real and imaginary parts, i.e.

$$
C_{Z}(z)=C_{\mathscr{R}\{Z\}, \mathscr{G}\{Z\}}(x, y)=P(\mathscr{R}\{Z\} \leq x, \mathscr{I}\{Z\} \leq y),
$$

where $P(\cdot)$ denotes the probability, $\mathscr{R}\{Z\}$ and $\mathscr{E}\{Z\}$ represent the real and imaginary parts of $Z$ respectively, and the pair of real numbers $(x, y)$ can be identified with a complex number $z=x+j y$, associating with $(x, y)$ and the complex number $z=x+j y$, the probability density function of $Z$ is defined as the joint probability density function of $(\mathscr{R}\{Z\} \mathscr{E}\{Z\})$, i.e.

$$
f_{Z}(z)=f_{\mathscr{R}\{Z\}, \mathscr{E}\{Z\}}(x, y) .
$$

A complex random variable is independent if

$$
f_{Z}(z)=f_{\mathcal{R}\{Z\}}(x) f_{\mathscr{G}\{Z\}}(y) \text {. }
$$

The distribution of a complex random vector

$$
\mathbf{Z}=\left(Z_{1}, Z_{2}, \ldots, Z_{n}\right)
$$

is specified by the joint distribution of real random vector $\left(\mathscr{R}\left\{Z_{1}\right\}, \ldots, \mathscr{R}\left\{Z_{n}\right\}, \mathscr{E}\left\{Z_{1}\right\}, \ldots, \mathscr{G}\left\{Z_{n}\right\}\right)$

$$
\begin{aligned}
\mathbf{C}_{\mathbf{Z}}(\mathbf{Z})= & P\left(\mathscr{R}\left\{Z_{1}\right\} \leq \mathscr{R}\left\{z_{1}\right\}, \ldots, \mathscr{R}\left\{Z_{n}\right\} \leq \mathscr{R}\left\{z_{n}\right\},\right. \\
& \left.\mathscr{E}\left\{Z_{1}\right\} \leq \mathscr{P}\left\{z_{1}\right\}, \ldots, \mathscr{E}\left\{Z_{n}\right\} \leq \mathscr{P}\left\{Z_{n}\right\}\right),
\end{aligned}
$$

where $\mathbf{z}=\left(z_{1}, z_{2}, \ldots, z_{n}\right)$ denotes the complex-valued vector with $z_{i}=x_{i}+j y_{i}, i=1,2, \ldots, n$. The probability density function of $\mathbf{Z}$ is defined in terms of the joint probability density function of $2 n$ real random variables $\mathscr{R}\left\{Z_{i}\right\}$ and $\mathscr{G}\left\{Z_{i}\right\}$, that is

$$
f_{\mathbf{Z}}(\mathbf{z})=f_{\mathcal{R}\left\{Z_{1}\right\}, \ldots, \mathfrak{R}\left\{Z_{n}\right\}, \mathscr{E}\left\{Z_{1}\right\}, \ldots, \mathscr{E}\left\{Z_{n}\right\}}\left(x_{1}, \ldots, x_{n}, y_{1}, \ldots, y_{n}\right)
$$

Thus, the complex random variables $\left\{Z_{i}\right\}$ are independent if

$$
f_{\mathbf{Z}}(\mathbf{z})=\prod_{i=1}^{n} f_{Z_{i}}\left(z_{i}\right)
$$

\section{System Model}

In this section, a quaternary DS-SSMA system with complex signature sequences over Rayleigh fading channel is described. As shown in Fig.1, there are $K$ simultaneous users that transmit data asynchronously. The channel inputs and outputs are complex waveforms. Both the modulator and the receiver employ complex processing.

\subsection{Transmitter model}

The $i$-th user's data signal can be expressed as

$$
b_{i}(t)=\sum_{l=-\infty}^{\infty} b_{l}^{(i)} p_{T}(t-l T)
$$

where the function $p_{T}(t)$ is the rectangular pulse of duration $T, b_{l}^{(i)} \in\{(1 / \sqrt{2)}( \pm 1, \pm j)\}$ denotes the $l$-th complex quaternary data value of the $i$-th user, and it is assumed to take values with equal probability and be independently complex uniform, i.e., $\mathscr{R}\left\{b_{l}^{(i)}\right\}$ and $\mathscr{E}\left\{b_{l}^{(i)}\right\}$ are independent and uniform with the proba- 
bility

$$
\left\{\begin{array}{l}
P\left\{\mathscr{R}\left\{b_{l}^{(i)}\right\}=1 / \sqrt{2}\right\}=P\left\{\mathscr{R}\left\{b_{l}^{(i)}\right\}=-1 / \sqrt{2}\right\}=0.5 ; \\
P\left\{\mathscr{E}\left\{b_{l}^{(i)}\right\}=1 / \sqrt{2}\right\}=P\left\{\mathscr{E}\left\{b_{l}^{(i)}\right\}=-1 / \sqrt{2}\right\}=0.5 ;
\end{array}\right.
$$

The complex spreading signal for the $i$-th user can be expressed as

$$
a_{i}(t)=\sum_{m=-\infty}^{\infty} a_{m}^{(i)} \psi\left(t-m T_{c}\right)
$$

where $a_{m}^{(i)} \in\{ \pm 1, \pm j\}$ denotes the $m$-th complex chip value of the $i$-th user, which is quadriphase or 4phase complex sequence element, and the complex spreading sequence $a^{(i)}=\left\{a_{m}^{(i)}\right\}$ has the period $N$. The function $\psi(t)$ is an arbitrary chip waveform that is time-limited to $\left[0, T_{c}\right)$, including the rectangular pulse, and $T_{c}$ is the chip duration. It is assumed that there is one period of the spreading sequence per data symbol, so $T=N T_{c}$. Thus, the $i$-th transmitted signal is described by

$$
s_{i}(t)=\sqrt{2 P_{i}} b_{i}(t) a_{i}(t) \exp \left\{j\left(\omega_{c} t+\theta_{i}\right)\right\},
$$

where $\sqrt{2 P_{i}} \exp \left\{j\left(\omega_{c} t+\theta_{i}\right)\right\}$ is the complex carrier signal, as shown in Fig. $1 ; P_{i}$ represents the $i$-th transm-

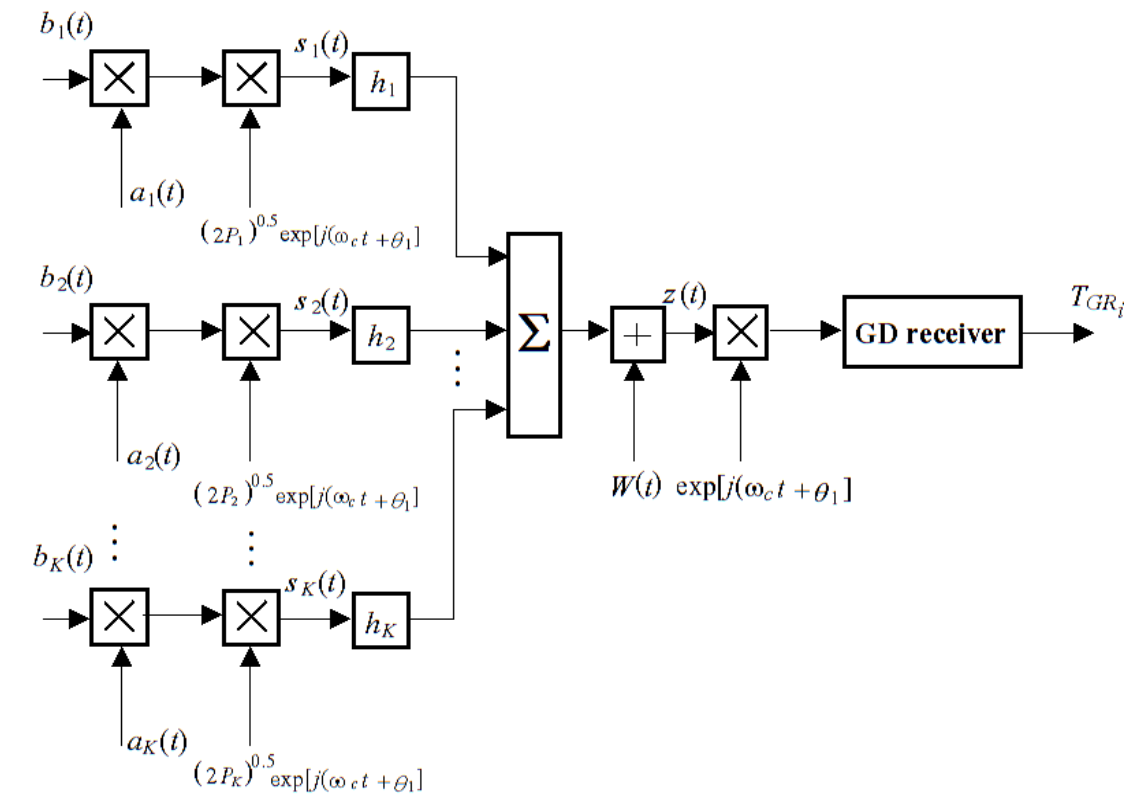

Fig. 1. DS-SSMA system with complex modulator and complex generalized receiver.

itted signal power; $\omega_{c}$ is the common complex carrier frequency; $\theta_{i}$ is the carrier phase of the $i$-th carrier, which is an independently uniform random variable within the limits of the interval $[0,2 \pi)$. Power control is assumed to be perfect, and the transmitted signal power $P_{i}$ is assumed to be known.

\subsection{Rayleigh fading channel model}

In Fig. 1, each signal $s_{i}(t)$ is transmitted over a frequency non-selective fading channel, where the user signals and the interfering signals all experience mutually independent Rayleigh fading. The fading is also assumed to be slow such that coherent detection is feasible. The channel impulse response for the $i$-th transmitted signal takes the form

$$
h_{i}(t)=A_{i} \exp \left\{j \beta_{i}\right\} \delta\left(t-\tau_{i}\right),
$$

where the fading random variables $A_{i}, 1 \leq i \leq K$, are independent, Rayleigh distributed and account for the fading channel attenuation of all signals. The fading random variables are assumed to have the same probability density function (pdf) and the pdf of $A_{i}$ is

$$
f_{A_{i}}(\alpha)= \begin{cases}\alpha \exp \left\{-0.5 \alpha^{2}\right\}, & \alpha \geq 0 \\ 0, & \alpha<0\end{cases}
$$

with $E\left[A_{i}^{2}\right]=2, E[\cdot]$ denotes the mathematical expectation. In (17), $\beta_{i}, 1 \leq i \leq K$ are the phases introduced by the fading channel and are assumed to be uniform within the limits of the interval $[0,2 \pi) ; \delta(t)$ is the Dirac impulse function, and $\tau_{i}, 1 \leq i \leq K$, are the time delays which are assumed to be uniform within the limits of the interval $[0, T)$ and independent.

The transmitted signal is further assumed to experience an additive background thermal band-pass noise process

$$
w(t)=r(t) \exp \left\{j \omega_{c} t\right\},
$$

where 


$$
r(t)=x(t)+j y(t),
$$

$x(t)$ and $y(t)$ are independent zero-mean baseband Gaussian noise processes, each having power spectral density given by $S(f)=0.25 \mathcal{N}_{0}^{\circ}$ for $|f| \leq 0.5 B$ and $S(f)=0$ for $|f|>0.5 B$, where $\mathcal{N}_{0}$ is the power spectral density of the white Gaussian noise. We assume that the bandwidth $B$ of the noise is much greater than the bandwidth of the baseband signals $b_{i}(t)$ and $a_{i}(t)$. The average power of the band-pass noise process is

$$
E\left[n^{2}(t)\right]=0.5 \mathcal{N}_{0} B .
$$

The received signal at the input of the generalized receiver takes the following form

where

$$
\begin{aligned}
z(t)= & \sum_{i=1}^{K} \sqrt{2 P_{i}} A_{i} b_{i}\left(t-\tau_{i}\right) a_{i}\left(t-\tau_{i}\right) \\
& \times \exp \left\{j\left(\omega_{c} t+\varphi_{i}\right)\right\}+w(t),
\end{aligned}
$$

$$
\varphi_{i}=\beta_{i}+\theta_{i}-\omega_{c} \tau_{i}
$$

All delays are defined by modulo $T$ and all carrier phase angles are defined by modulo $2 \pi$. This allows one to restrict attention to $0 \leq \tau_{i}<T, 0 \leq \varphi_{i}<2 \pi, \forall i$. It is easy to show that $\tau_{i}$ and $\varphi_{i}$ can be modelled as mutually independent uniform random variables for very large $\omega_{c}$. Hence, in this paper, it is further assumed that all $A_{i}, \varphi_{i}, \tau_{i}$ are mutually independent random variables.

\subsection{Generalized receiver}

To coherently demodulate the desired user signal in an asynchronous system the conventional generalized receiver (see Fig.2) is employed by DS-SSMA system. As we mentioned before, the generalized receiver is constructed in accordance with the generalized approach to signal processing in noise [13] [15]. The generalized approach to signal processing introduces an additional noise source that does not carry any information about the parameters of desired transmitted signal with the purpose to improve the signal processing system performance. This additional noise can be considered as the reference noise without any information about the parameters of the signal to be detected.

The jointly sufficient statistics of the mean and variance of the likelihood function is obtained under the generalized approach to signal processing in noise employment, while the classical and modern signal processing theories can deliver only a sufficient statistics of the mean or variance of the likelihood function. Thus, the generalized approach to signal processing in noise implementation allows us to ob- tain more information about the desired transmitted signal incoming at the generalized receiver input. Owing to this fact, the detectors constructed based on the generalized approach to signal processing in noise technology are able to improve the signal detection performance in comparison with other conventional detectors.

The generalized receiver (GR) consists of three channels (see Fig.2): the GR correlation detector channel (GR CD) - the preliminary filter (PF), the multipliers 1 and 2, the model signal generator (MSG); the GR energy detector channel (GR ED) the PF, the additional filter (AF), the multipliers 3 and 4 , the summator 1 ; and the GR compensation channel (GR CC) - the summators 2 and 3, the accumulator 1. The threshold apparatus (THRA) device defines the GR threshold. As we can see from Fig.2, there are two band-pass filters, i.e. the linear systems, at the GR input, namely, the PF and AF. We assume for simplicity that these two filters or linear systems have the same amplitude-frequency characteristics or impulse responses. The AF central frequency is detuned relative to the PF central frequency.

There is a need to note the PF bandwidth is matched with the bandwidth of the transmitted signal bandwidth. If the detuning value between the PF and $\mathrm{AF}$ central frequencies is more than 4 or 5 times the transmitted signal bandwidth to be detected, i.e. $4 \div$ $5 \Delta f_{s}$, where $\Delta f_{s}$ is the transmitted signal bandwidth, we can believe that the processes at the $\mathrm{PF}$ and $\mathrm{AF}$ outputs are uncorrelated because the coefficient of correlation between them is negligible (not more than 0.05). This fact was confirmed experimentally in [26] and [27] independently.

Thus, the transmitted signal plus noise can be appeared at the GR PF output and the noise only is appeared at the GR AF output. The stochastic processes at the AF and PF outputs present the input stochastic samples from two independent frequency-time regions. If the discrete-time noise $w_{i}[k]$ at the PF and $\mathrm{AF}$ inputs is Gaussian, the discrete-time noise $\zeta_{i}[k]$ at the PF output is Gaussian and the reference discrete-time noise $\eta_{i}[k]$ at the AF output is Gaussian, too, owing to the fact that the PF and AF are the linear systems and we believe that these linear systems do not change the statistical parameters of the input process.

Thus, the AF can be considered as a generator of the reference noise with a priori information a "no" transmitted signal (the reference noise sample) [15, Chapter 5]. The noise at the PF and AF outputs can be presented as 


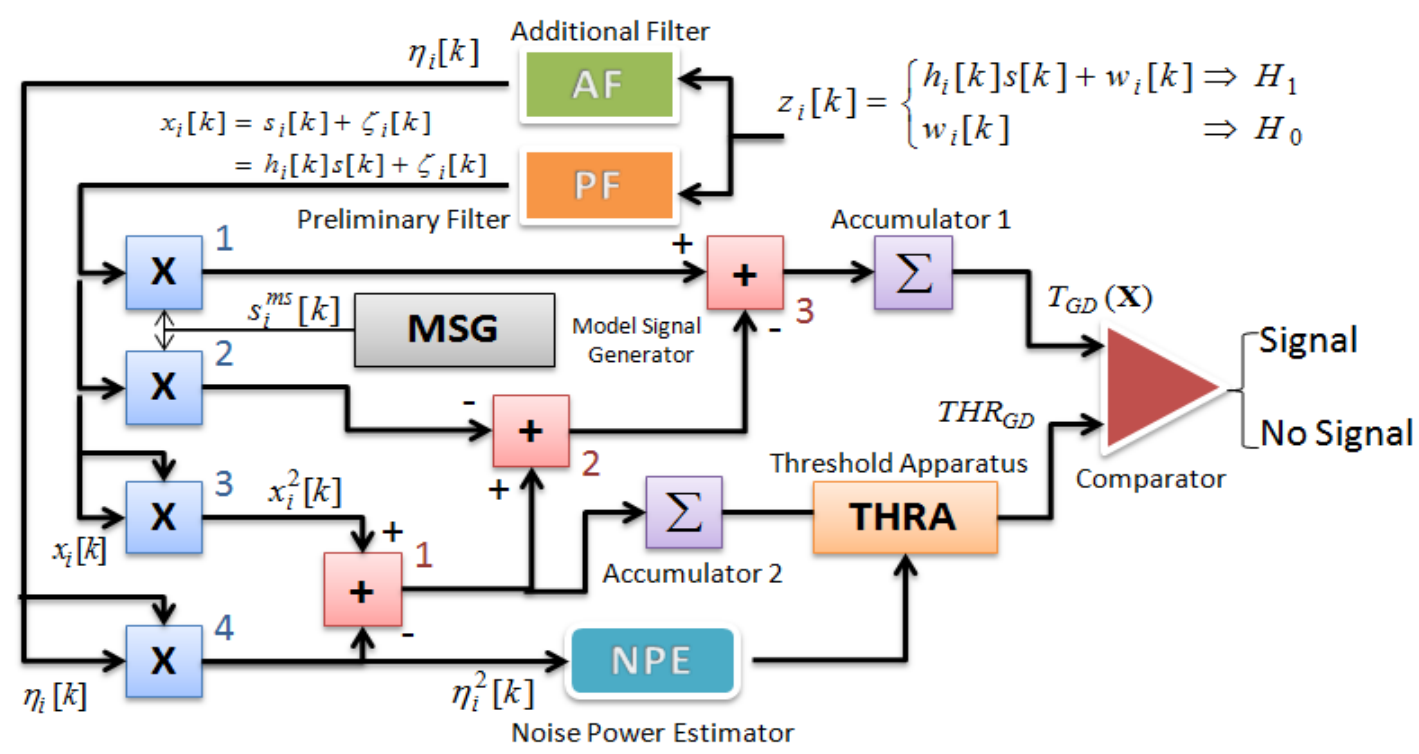

Fig. 2. Generalized receiver structure.

$$
\left\{\begin{array}{l}
\zeta_{i}[k]=\sum_{m=-\infty}^{\infty} g_{P F}[m] w_{i}[k-m] ; \\
\eta_{i}[k]=\sum_{m=-\infty}^{\infty} g_{A F}[m] w_{i}[k-m],
\end{array}\right.
$$

where $g_{P F}[m]$ and $g_{A F}[m]$ are the impulse responses of the PF and AF, respectively.

In a general, under practical implementation of any detector in communication system with sensor array, the bandwidth of the spectrum to be sensed is defined. Thus, the AF bandwidth and central frequency can be assigned, too (the AF bandwidth can not be used by the transmitted signal because it is out of its spectrum). The case when there are interfering signals within the AF bandwidth, the action of this interference on the GR detection performance, and the case of non-ideal condition when the noise at the PF and AF outputs is not the same by statistical parameters are discussed in [28] and [29].

Under the hypothesis $\mathscr{H}_{l}$ ("a yes" transmitted signal), the GR CD generates the signal component $s_{i}^{m}[k] s_{i}[k]$ caused by interaction between the model signal $s_{i}^{m}[k]$, the MSG output, and the incoming signal $s_{i}[k]$, and the noise component $s_{i}^{m}[k] \zeta_{i}[k]$ caused by interaction between the model signal $s_{i}^{m}[k]$ and the noise $\zeta_{i}[k]$ at the PF output. GR ED generates the transmitted signal energy $s_{i}^{2}[k]$ and the random component $s_{i}[k] \zeta_{i}[k]$ caused by interaction between the transmitted signal $s_{i}[k]$ and the noise $\zeta_{i}[k]$ at the PF output. The main purpose of the GR CC is to cancel completely in the statistical sense the GR CD noise component $s_{i}^{m}[k] \zeta_{i}[k]$ and the GR ED rand- om component $s_{i}[k] \zeta_{i}[k]$ based on the same nature of the noise $\zeta_{i}[k]$. The relation between the transmitted signal to be detected $s_{i}[k]$ and the model signal $s_{i}^{m}[k]$ is defined as:

$$
s_{i}^{m}[k]=\mu s_{i}[k],
$$

where $\mu$ is the coefficient of proportionality.

The main functioning condition under the GR employment in any signal processing system including the communication one with radar sensors is the equality between the parameters of the model signal $s_{i}^{m}[k]$ and the incoming signal $s_{i}[k]$, for example, by amplitude. Under this condition it is possible to cancel completely in the statistical sense the noise component $s_{i}^{m}[k] \zeta_{i}[k]$ of the GR CD and the random component $s_{i}[k] \zeta_{i}[k]$ of the GR ED. Satisfying the GR main functioning condition given by $(25), s_{i}^{m}[k]=$ $s_{i}[k], \mu=1$, we are able to detect the transmitted signal with the high probability of detection at the low signal-to-noise ratio $(S N R)$ and define the transmitted signal parameters with high accuracy.

Practical realization of this condition (25) at $\mu \rightarrow$ 1 requires increasing in the complexity of GR structure and, consequently, leads us to increasing in computation cost. For example, there is a need to employ the amplitude tracking system or to use the offline data samples processing. Under the hypothesis $\mathscr{H}_{0}$ ("a no" transmitted signal), satisfying the main GR functioning condition (25) at $\mu \rightarrow 1$ we obtain only the back-ground noise $\eta_{i}^{2}[k]-\zeta_{i}^{2}[k]$ at the GR output. 
Under practical implementation, the real structure of GR depends on specificity of signal processing systems and their applications, for example, the radar sensor systems, adaptive communication systems, cognitive radio systems, satellite communication systems, mobile communication systems and so on. In the present paper, the GR circuitry (Fig.2) is demonstrated with the purpose to explain the main functioning principles. Because of this, the GR flowchart presented in the paper should be considered under this viewpoint. Satisfying the GR main functioning condition (25) at $\mu \rightarrow 1$, the ideal case, for communication systems with radar sensor applications we are able to detect the transmitted signal with very high probability of detection and define accurately its parameters.

In the present paper, we discuss the GR implementation in communication systems using the radar sensor array. Since the presented GR test statistics is defined by the signal energy and noise power, the equality between the model signal $s_{i}^{m}[k]$ and the transmitted signal to be detected $s_{i}[k]$, in particular by amplitude, is required that leads us to high circuitry complexity in practice. For example, there is a need to employ the amplitude tracking system or off-line data sample processing. Detailed discussion about the main GR functioning principles if there is no a priori information and there is an uncertainty about the parameters of transmitted signal, i.e., the transmitted signal parameters are random, can be found in [13] and [14, Chapter 6, pp.611-621 and Chapter 7, pp. 631-695].

The complete matching between the model signal $s_{i}^{m}[k]$ and the incoming signal $s_{i}[k]$, for example by amplitude, is a very hard problem in practice because the incoming target return signal $s_{i}[k]$ depends on both the fading and the transmitted signal parameters and it is impractical to estimate the fading gain at the low $S N R$. This matching is possible in the ideal case only. The GD detection performance will be deteriorated under mismatching in parameters between the model signal $s_{i}^{m}[k]$ and the transmitted signal $s_{i}[k]$ and the impact of this problem is discussed in [18] and [30], where a complete analysis about the violation of the main GR functioning requirements is presented. The GR decision statistics requires an estimation of the noise variance $\sigma_{\eta}^{2}$ using the reference noise $\eta_{i}[k]$ at the AF output.

Under the hypothesis $\mathscr{\not}_{1}$, the signal at the PF output, see Fig. 2, can be defined as

$$
x_{i}[k]=s_{i}[k]+\zeta_{i}[k],
$$

where $\zeta_{i}[k]$ is the noise at the PF output and

$$
s_{i}[k]=h_{i}[k] s[k],
$$

where $h_{i}[k]$ are the channel coefficients. Under the hypothesis $\mathscr{H}_{0}$ and for all $i$ and $k$, the process $x_{i}[k]=$ $\zeta_{i}[k]$ at the PF output is subjected to the complex Gaussian distribution and can be considered as the independent and identically distributed (i.i.d.) process.

In ideal case, we can think that the signal at the AF output is the reference noise $\eta_{i}[k]$ with the same statistical parameters as the noise $\zeta_{i}[k]$. In practice, there is a difference between the statistical parameters of the noise $\eta_{i}[k]$ and $\zeta_{i}[k]$. How this difference impacts on the GR detection performance is discussed in detail in [14, Chapter 7, pp. 631-695] and in [18] and [30].

The decision statistics at the GR output presented in [13] and [14, Chapter 3] is extended for the case of antenna array when an adoption of multiple antennas and antenna arrays is effective to mitigate the negative attenuation and fading effects. The GR decision statistics can be presented in the following form:

$$
\begin{gathered}
T_{G R}(\mathbf{X})=\sum_{k=0}^{N-1} \sum_{i=1}^{M} 2 x_{i}[k] s_{i}^{m}[k] \\
-\sum_{k=0}^{N-1} \sum_{i=1}^{M} x_{i}^{2}[k]+\sum_{k=0}^{N-1} \sum_{i=1}^{M} \eta_{i}^{2}[k] \stackrel{\mathscr{H}_{0}}{\underset{\mathcal{H}_{1}}{>}} T H R_{G R},
\end{gathered}
$$

where

$$
\mathbf{X}=[\mathbf{x}(0), \ldots, \mathbf{x}(N-1)]
$$

is the vector of the random process at the PF output and $T H R_{G R}$ is the GR detection threshold.

Under the hypotheses $\mathscr{H}_{1}$ and $\mathscr{H}_{0}$ and when the amplitude of the transmitted signal is equal to the amplitude of the model signal, $s_{i}^{m}[k]=s_{i}[k]$, the GR decision statistics $T_{G D}(\mathbf{X})$ takes the following form in the statistical sense, respectively:

$$
\left\{\begin{aligned}
\mathscr{H}_{1}: T_{G D}(\mathbf{X}) & =\sum_{k=0}^{N-1} \sum_{i=1}^{M} s_{i}^{2}[k] \\
& +\sum_{k=0}^{N-1} \sum_{i=1}^{M} \eta_{i}^{2}[k]-\sum_{k=0}^{N-1} \sum_{i=1}^{M} \zeta_{i}^{2}[k], \\
\mathscr{H}_{0}: T_{G D}(\mathbf{X}) & =\sum_{k=0}^{N-1} \sum_{i=1}^{M} \eta_{i}^{2}[k]-\sum_{k=0}^{N-1} \sum_{i=1}^{M} \zeta_{i}^{2}[k] .
\end{aligned}\right.
$$

In (30) the term $\sum_{k=0}^{N-1} \sum_{i=1}^{M} s_{i}^{2}[k]=E_{s}$ corresponds to the average transmitted signal energy, and the 
term $\sum_{k=0}^{N-1} \sum_{i=1}^{M} \eta_{i}^{2}[k]-\sum_{k=0}^{N-1} \sum_{i=1}^{M} \zeta_{i}^{2}[k]$ is the background noise at the GR output. The GR output background noise is a difference between the noise power at the PF and AF outputs. Practical implementation of the GR decision statistics requires an estimation of the noise variance $\sigma_{\eta}^{2}$ using the reference noise $\eta_{i}[k]$ at the AF output.

\subsection{Generalized receiver in DS-SSMA system}

Fig.1 represents a conventional generalized receiver, a block diagram of which is demonstrated in Fig.2, to coherently demodulate the desired user signal in an asynchronous system. It should be noted that each user signal undergoes a Rayleigh flat-fading channel, thus, an equalizer is not included in the receiver. By symmetry, mathematical derivation of the average error probability is the same for all users. We assume, without loss of generality, the $i$-th user is the target one and $\tau_{i}=\varphi_{i}=0$. Hence, all delays and carrier phase shifts are measured relative to those of the $i$-th signal. The decision statistic is the output of a complex generalized receiver that employs the complex carrier $\exp \left\{-j \omega_{c} t\right\}$ and the complex spreading signal $a_{i}^{*}(t)$.

The output of the complex generalized receiver matched to the $i$-th signal is the random variable given by

$$
T_{G R_{i}}=\int_{0}^{T}\left[2 z(t) a_{i}^{*}(t)-z^{2}(t)+\eta^{2}(t)\right] \exp \left\{-j \omega_{c} t\right\} d t .
$$

Taking into consideration the general ideal condition of functioning of the generalized receiver $\mu \rightarrow 1$ and $s_{i}^{m s}[k]=s_{i}[k]$, we can rewrite (31) in the following form:

$$
T_{G R_{i}}=I_{i}(b, \tau, \varphi)+\sqrt{2 P_{i}} A_{i} b_{0}^{(i)} T+\varsigma_{i}(t),
$$

where $\varsigma_{i}^{2}(t)$ is the background noise defined as

$$
\varsigma_{i}(t)=\int_{0}^{T}\left[\eta_{i}^{2}(t)-\zeta_{i}^{2}(t)\right] d t
$$

and $I_{i}(b, \tau, \varphi)$ is the total multiple-access interference (MAI) defined as

$$
\begin{aligned}
I_{i}(\bar{b}, \bar{\tau}, \bar{\varphi}) & =\sum_{i=1, i \neq k}^{K} \sqrt{2 P_{i}} A_{i} \exp \left\{j \varphi_{i}\right\} \\
& \times\left[b_{-1}^{(i)} R_{i, k}\left(\tau_{i}\right)+b_{0}^{(i)} \hat{R}_{i, k}\left(\tau_{i}\right)\right]
\end{aligned}
$$

with

$$
\left\{\begin{array}{l}
b^{(i)}=\left(b_{-1}^{(i)}, b_{0}^{(i)}\right) ; \\
\bar{b}=\left(b^{(1)}, b^{(2)}, \ldots, b^{(i-1)}, b^{(i+1)}, \ldots, b^{(K)}\right) ; \\
\bar{\tau}=\left(\tau_{1}, \tau_{2}, \ldots, \tau_{i-1}, \tau_{i+1}, \ldots \tau_{K}\right) ; \\
\bar{\varphi}=\left(\varphi_{1}, \varphi_{2}, \ldots, \varphi_{i-1}, \varphi_{i+1}, \ldots \varphi_{K}\right)
\end{array}\right.
$$

$$
\left\{\begin{array}{l}
R_{i, k}\left(\tau_{i}\right)=\int_{0}^{\tau} a_{i}(t-\tau) a_{k}^{*}(t) d t \\
\hat{R}_{i, k}\left(\tau_{i}\right)=\int_{\tau}^{T} a_{i}(t-\tau) a_{k}^{*}(t) d t
\end{array}\right.
$$

for $0 \leq \tau \leq T$.Along the lines of [4], it is easy to show that for $0 \leq \tau \leq T$, these two cross correlation functions can be written in the following form:

$$
\left\{\begin{aligned}
R_{i, k}\left(\tau_{i}\right)= & C_{i, k}(l-N) \hat{R}_{\psi}\left(\tau-l T_{c}\right)+C_{i, k}(l+1-N) \\
& \times R_{\psi}\left(\tau-l T_{c}\right), \\
\hat{R}_{i, k}\left(\tau_{i}\right)= & C_{i, k}(l) \hat{R}_{\psi}\left(\tau-l T_{c}\right)+C_{i, k}(l+1) R_{\psi}\left(\tau-l T_{c}\right),
\end{aligned}\right.
$$

where $l=\left\lfloor\tau / T_{c}\right\rfloor$ is the integer part of $\tau / T_{c} ; R_{\psi}(s)$ and $\hat{R}_{\psi}(s)$ are the continuous-time partial autocorrelation functions of the chip waveform defined as

$$
\left\{\begin{array}{l}
R_{\psi}(s)=\int_{0}^{s} \psi(t) \psi\left(t+T_{c}-s\right) d t, \\
\hat{R}_{\psi}(s)=\int_{s}^{T_{c}} \psi(t) \psi(t-s) d t
\end{array}\right.
$$

for $0 \leq s \leq T_{c}$ and zero otherwise. Therefore, the multiple-access interference (34) can be presented in the following form:

$$
\begin{gathered}
I_{i}(b, \tau, \varphi)=\sum_{i=1, i \neq k}^{K} \sqrt{2 P_{i}} A_{i} \exp \left\{j \varphi_{i}\right\} b_{0}^{(i)} \\
\times\left\{\left[b_{-1}^{(i)}\left(b_{0}^{(i)}\right)^{*} C_{i, k}\left(l_{i}-N\right)+C_{i, k}\left(l_{i}\right)\right] \hat{R}_{\psi}\left(\tau_{i}-l_{i} T_{c}\right)\right. \\
\left.+\left[b_{-1}^{(i)}\left(b_{0}^{(i)}\right)^{*} C_{i, k}\left(l_{i}+1-N\right)+C_{i, k}\left(l_{i}+1\right)\right] R_{\psi}\left(\tau_{i}-l_{i} T_{c}\right)\right\} \\
=\sum_{i=1, i \neq k}^{K} \sqrt{2 P_{i}} A_{i} \exp \left\{j \varphi_{i}\right\} b_{0}^{(i)}\left[\theta_{i, k}^{(n)}\left(l_{i}\right) \hat{R}_{\psi}\left(\tau_{i}-l_{i} T_{c}\right)\right. \\
\left.+\theta_{i, k}^{(n)}\left(l_{i}+1\right) R_{\psi}\left(\tau_{i}-l_{i} T_{c}\right)\right]
\end{gathered}
$$

where $l=\left\lfloor\tau / T_{c}\right\rfloor, n \in\{0,1,2,3\}$ satisfies $\exp \{j \pi n / 2\}=$ $b_{-1}^{(i)}\left(b_{0}^{(i)}\right)^{*}$ and $\theta_{i, k}^{(n)}(l)$ is the complex quaternary correlation function defined in [31] as

$$
\theta_{i, k}^{(n)}(l)=C_{i, k}(l)+C_{i, k}(l-N) \exp \{j \pi n / 2\}
$$

with $0 \leq l \leq N-1$. Obviously, $\theta_{i, k}^{(0)}(l)$ is the complex quaternary periodic cross correlation function and $\theta_{i, k}^{(n)}(l), n=1,2,3$ is the quaternary odd cross correlation function.

\section{System Performance}

In this section, the exact formulas for the average bit error rate $(B E R)$ for the synchronous system with quaternary signal and complex sequences over Rayleigh fading channel is derived first. Thereafter, the average $B E R$ for quaternary asynchronous system with quaternary signal and complex sequences over 
Rayleigh fading channel is evaluated using the characteristic function approach. The Gaussian approximate average $B E R$ for the asynchronous system under consideration is obtained with the MAI being modeled as a complex Gaussian random variable. A symbol error occurs in the complex processing system if the decision statistic $T_{G D_{i}}(\mathbf{X})$ is not in the same quadrant of the complex plane as the data symbol from the desired signal. Since $b_{l}^{(i)} \in\{(1 / \sqrt{2})( \pm 1 \pm j)\}$ are the quaternary phase data, the method in [32] can be adopted with some modifications to include the interference action. Thus, simple computations show that for Gray-coded quaternary signal transmission system with complex sequences in the flat Rayleigh fading, the average $B E R$ of the $i$-th Rayleigh faded user is given by

$$
B E R=0.5\left[E\left(P_{i \Re}\right)+E\left(P_{i \mathscr{G}}\right)\right],
$$

where $P_{i \Re}$ is the average $B E R$ that $\mathscr{R}\left\{T_{G R_{i}}\right\}$ is not of the same polarity as $\mathscr{R}\left\{b_{0}^{(i)}\right\}= \pm 1 / \sqrt{2}$ and $P_{i \mathscr{g}}$ is the average probability that $\mathscr{E}\left\{T_{G R_{i}}\right\}$ is not of the same polarity as $\mathscr{G}\left\{b_{0}^{(i)}\right\}= \pm 1 / \sqrt{2}$. The expectation operator $E$ is taken over the random vectors $\mathbf{b}, \boldsymbol{\tau}, \varphi$ and random variable $A_{i}$, which are assumed to be mutually independent.

\subsection{Synchronous BER Analysis}

In this subsection, we consider the $B E R$ calculation for a synchronous system, i.e., $\tau_{1}=\tau_{2}=\ldots=\tau_{K}=0$ in (22). The exact BER for the synchronous system with complex spreading sequences in Rayleigh fading is derived here. The presented derivation both serves completeness and clarifies the derivation and understanding of the asynchronous results in the next subsection. Let us describe the BER property of the synchronous system under consideration. We consider the synchronous DS-SSMA system with complex signature sequences and complex generalized receiver over flat Rayleigh fading channel presented in Fig.1. Then the average synchronous BER for a Rayleigh-faded user is

$$
\overline{B E R}=0.5\left[\overline{B E R}_{i} R+\overline{B E R}_{i \mathcal{G}}\right]=0.5\left(1-\Delta_{s}\right),
$$

where $E_{i}=P_{i} T$ is the energy per data symbol and

$$
\Delta_{s}=\frac{1}{\sqrt{1+\sum_{i=1, i \neq k}^{K} \frac{2 P_{i}}{N^{2} P_{k}} C_{i, k}(0) C_{i, k}^{*}(0)+\frac{N_{0}^{2}}{16 E_{i}^{2}}}} .
$$

Let us show it. The output of the complex generalized receiver matched to the $i$-th signal is given by (31) and (32), where $\varsigma_{i}(t)=\varsigma_{i \Re}(t)+\varsigma_{i \mathscr{G}}(t)$ is as (33) with $\varsigma_{i \Re}(t)$ and $\varsigma_{i \mathscr{G}}(t)$ being independent random va- riable distributed according to the modified second kind Bessel function of an imaginary argument or, as it also called, McDonald function each having the mean $\sigma_{\eta_{i R}}^{2}=\sigma_{\zeta_{i g}}^{2}=N_{0} T / 4$ and variance $\frac{1}{16} N_{0}^{2} T^{2}$ in the simplest ideal case when $\sigma_{\eta}^{2}=\sigma_{\zeta}^{2}$, i.e., the power of the noise at the $\mathrm{PF}$ and $\mathrm{AF}$ of the generalized receiver are equal between each other. In the statistical sense we can assume that $\varsigma_{i}(t)$ can be considered as the random process with asymptotic Gaussian distribution with the mean $\sigma_{\eta_{i R}}^{2}=\sigma_{\zeta_{i g}}^{2}=N_{0} T / 4$ and variance $(1 / 16) N_{0}^{2} T^{2}$.

In view of (37) and (38)

$$
\begin{aligned}
I_{i}(b, \tau, \varphi) & =\sum_{i=1, i \neq k}^{K} \sqrt{2 P_{i}} A_{i} \exp \left\{j \varphi_{i}\right\} b_{0}^{(i)} C_{i, k}(0) T_{c} \\
& =\sum_{i=1, i \neq k}^{K} \sqrt{2 P_{i}} D_{i} b_{0}^{(i)} C_{i, k}(0) \frac{T}{N},
\end{aligned}
$$

where $D_{i}=A_{i} \exp \left\{j \varphi_{i}\right\}=D_{i_{1}}+j D_{i_{2}}$ is the zero-mean complex Gaussian variable with the unit variance, i.e., $D_{i_{1}}$ and $D_{i_{2}}$ are the independent zero-mean real Gaussian variables with unit variance. This is true since $A_{i}$ is the Rayleigh distributed; $\varphi_{i}$ is the uniformly distributed within the limits of the interval $[0$, $2 \pi$ ) and $A_{i}$ and $\varphi_{i}$ are independent; $b_{0}^{(i)}$ takes the values within the limits of the interval $\{(1 / \sqrt{2})( \pm 1 \pm j)\}$ with independent complex uniform, and is independent of $D_{i}$, so $D_{i}^{\prime}=D_{i} b_{0}^{(i)}$ is the independent zero-mean complex Gaussian variable with the unit variance. Note that $\left\{A_{i}, \varphi_{i}, b_{0}^{(i)}\right\}_{i=1}^{K}$ are mutually independent complex random variables, hence, $I_{i}$ is the complex Gaussian random variable with the real and imaginary parts being zero-mean real independent Gaussian random variables and each having variance

$$
\begin{aligned}
\sigma_{I, \mathscr{R}}^{2} & =\sigma_{I, \mathscr{E}}^{2}=\sum_{i=1, i \neq k}^{K} \frac{2 P_{i} T^{2}}{N^{2}}\left|C_{i, k}(0)\right|^{2} \\
& =\sum_{i=1, i \neq k}^{K} \frac{2 P_{i} T^{2}}{N^{2}} C_{i, k}(0) C_{i, k}^{*}(0) .
\end{aligned}
$$

Define

$$
I_{i}^{\prime}=I_{i}(b, \tau, \varphi)+\varsigma_{i}(t)=I_{i_{1}}^{\prime}+j I_{i_{2}}^{\prime},
$$

with $I_{i_{1}}^{\prime}$ and $I_{i_{2}}^{\prime}$ being the real and imaginary parts of $I_{i}^{\prime}$. Then it follows from (44) and (45) that $I_{i_{1}}^{\prime}$ and $I_{i_{2}}^{\prime}$ are the independent asymptotic zero-mean Gaussian random variables with the variances $\sigma_{I_{i} R}^{2}+\sigma_{S_{i} R}^{2}$ and $\sigma_{I_{i} \mathscr{G}}^{2}+\sigma_{\varsigma_{i} \mathscr{C}}^{2}$, respectively, i.e., $I_{i}^{\prime}$ is the complex Gaussian random variable. By symmetry and using the independence of $I_{i}$ and $\varsigma_{i}$, given $A_{i}$, the average co- 
nditional $B E R$ s for the real and imaginary parts of the $i$-th user are

$$
=Q\left\{\begin{array}{c}
\overline{B E R}_{i \mathscr{} \mid A_{i}}=\overline{B E R}_{i \mathscr{Q} \mid A_{i}} \\
\sqrt{P_{i} A_{i} T} \\
\sqrt{\sum_{i=1, i \neq k}^{K} \frac{2 P_{i} T^{2}}{N^{2}} C_{i, k}(0) C_{i, k}^{*}(0)+\frac{N_{0}^{2} T^{2}}{16}}
\end{array},\right.
$$

where

$$
Q(x)=\frac{1}{2 \pi} \int_{x}^{\infty} \exp \left\{-0.5 t^{2}\right\} d t .
$$

Averaging over $A_{i}$ with respect to the Rayleigh distribution in (18) and using the integral identity

$$
\int_{0}^{\infty} x \exp \left\{-0.5 x^{2}\right\} Q(x / \sigma) d x=\frac{1}{2}-\frac{1}{2 \sqrt{\sigma^{2}+1}},
$$

the average synchronous $B E R$ for a Rayleigh-faded user is obtained in (42). Note that UCHT sequences are orthogonal, i.e., $C_{i, k}(0)=0, i \neq k ; \Delta_{s}$ in (42) for quaternary synchronous systems with complex UCHT sequences reduces to

$$
\Delta_{s}=1+\left(\sqrt{\frac{N_{0}^{2}}{16 E_{i}^{2}}}\right)^{-1}=1+\frac{4 E_{i}}{N_{0}} .
$$

This shows that the output of the $i$-th generalized receiver is not affected at all by the signals of other users, and it is only affected by the background noise and the Rayleigh fading.

\subsection{Asynchronous BER Analysis}

In this subsection, the average $B E R$ calculation for asynchronous DS-SSMA system with complex signature sequences is investigated. The channel is assumed to be the Rayleigh fading and complex processing is adopted for the modulator and generalized receiver.

\subsubsection{Characteristic Function Approach}

\section{Define}

$$
d_{i, k}^{(n)}=\theta_{i, k}^{(n)}\left[\left(l_{i}\right) \hat{R}_{\psi}\left(\tau_{i}-l_{i} T_{c}\right)+\left(l_{i}+1\right) R_{\psi}\left(\tau_{i}-l_{i} T_{c}\right),\right.
$$

where $n=0,1,2,3$ and

$$
I_{i, k}=\sqrt{2 P_{i}} D_{i} b_{0}^{(i)} d_{i, k}^{(n)} .
$$

Then, (39) can be written in the following form:

$$
I_{i}(b, \tau, \varphi)=\sum_{i=1, i \neq k}^{K} I_{i, k}=\sum_{i=1, i \neq k}^{K} \sqrt{2 P_{i}} D_{i} b_{0}^{(n)} d_{i, k}^{(n)} .
$$

Since

$$
D_{i}=A_{i} \exp \left\{j \varphi_{i}\right\}=D_{i_{1}}+j D_{i_{2}}
$$

is the independent zero-mean complex Gaussian with the unit variance, then, given $b^{(i)}=\left(b_{0}^{(i)}, b_{-1}^{(i)}\right)$ and $\tau_{i}$

$$
I_{i, k}=I_{i, k, R}+j I_{i, k, \mathscr{E}}
$$

is the independent zero-mean complex Gaussian with the variance

$$
\sigma_{i, k, R}^{2}=\sigma_{i, k, \mathscr{g}}^{2}=2 P_{i}\left|d_{i, k}^{(n)}\right|^{2} .
$$

This implies that the conditional probability density function for $I_{i, k}$ takes the following form:

$$
f_{I_{i, k} \mid b^{(i)}, \tau_{i}}(x, y)=\frac{1}{4 P_{i} \pi\left|d_{i, k}^{(n)}\right|^{2}} \exp \left\{-\frac{x^{2}+y^{2}}{4 P_{i}\left|d_{i, k}^{(n)}\right|^{2}}\right\} .
$$

Note that

$$
b^{(i)}=\left(b_{0}^{(i)}, b_{-1}^{(i)}\right) \in\{(1 / \sqrt{2})( \pm 1 \pm j)\}^{2},
$$

averaging over $b^{(i)}$, we obtain

$$
\begin{gathered}
f_{I_{i, k} \mid b^{(i)}, \tau_{i}}(x, y) \\
=\frac{1}{4} \sum_{n=0}^{3} \frac{1}{4 P_{i} \pi\left|d_{i, k}^{(n)}\right|^{2}} \exp \left\{-\frac{x^{2}+y^{2}}{4 P_{i}\left|d_{i, k}^{(n)}\right|^{2}}\right\} .
\end{gathered}
$$

Since $\left|d_{i, k}^{(n)}\right|^{2}, n=0,1,2,3$ appears in the denominators of the exponential function arguments, thus it is difficult to average over $\tau_{i}$. In order to solve this, we employ the characteristic function approach. The characteristic function of $I_{i, k}$, given $\tau_{i}$, takes the following form:

$$
\Theta_{I_{i, k} \mid \tau_{i}}\left(\omega_{1}, \omega_{2}\right)=\frac{1}{4} \sum_{n=0}^{3} \exp \left\{-\left(\omega_{1}^{2}+\omega_{2}^{2}\right) P_{i}\left|d_{i, k}^{(n)}\right|^{2}\right\} .
$$

Averaging over $\tau_{i}$, the characteristic function of $I_{i, k}$ takes the form:

$$
\begin{gathered}
\Theta_{I_{i, k} \mid \tau_{i}}\left(\omega_{1}, \omega_{2}\right) \\
=\frac{1}{4} \sum_{n=0}^{3} \int_{0}^{T} \exp \left\{-\left(\omega_{1}^{2}+\omega_{2}^{2}\right) P_{i}\left|d_{i, k}^{(n)}\right|^{2}\right\} d \tau_{i} \\
=\frac{1}{4} \sum_{n=0}^{3} \sum_{l_{i}=0}^{N-1} \int_{l_{i}}^{\left(l_{i}+1\right) T_{c}} \exp \left\{-\left(\omega_{1}^{2}+\omega_{2}^{2}\right) P_{i}\left|d_{i, k}^{(n)}\right|^{2}\right\} d \tau_{i} .
\end{gathered}
$$

If the chip waveform $\psi(t)$ is the rectangular pulse of duration $T_{c}$, i.e., $\psi(t)=1$ for $0 \leq t<T_{c}$ and $\psi(t)=0$ otherwise. Then for this waveform, $R_{\psi}(\tau)=\tau$ and $\hat{R}_{\psi}(\tau)$ $=T_{c}-\tau$. Let

$$
u_{i}=\frac{\tau_{i}-l_{i} T_{c}}{T_{c}},
$$

so $u_{i} \in[0,1)$. Then (61) reduces to

$$
\begin{gathered}
\Theta_{I_{i, k} \mid \tau_{i}}\left(\omega_{1}, \omega_{2}\right) \\
=\frac{1}{4 N} \sum_{n=0}^{3} \sum_{l_{i}=0}^{N-1} \int_{0}^{1} \exp \left\{-\left(\omega_{1}^{2}+\omega_{2}^{2}\right) P_{i} T_{c}^{2}\right. \\
\left.\times\left|\theta_{i, k}^{(n)}\left(l_{i}\right)\left(1-u_{i}\right)+\theta_{i, k}^{(n)}\left(l_{i}+1\right) u_{i}\right|^{2}\right\} d u_{i} \\
\cong \frac{1}{4 N} \sum_{n=0}^{3} \sum_{l_{i}=0}^{N-1} \int_{0}^{1} \exp \left\{-\left(\omega_{1}^{2}+\omega_{2}^{2}\right) P_{i} T_{c}^{2} \mathscr{Z}_{i, k}^{(n)}\right\} d u_{i},
\end{gathered}
$$


where

$$
\mathscr{L}_{i, k}^{(n)}=\left|\theta_{i, k}^{(n)}\left(l_{i}\right)\left(1-u_{i}\right)+\theta_{i, k}^{(n)}\left(l_{i}+1\right) u_{i}\right|^{2} .
$$

In order to calculate $\Theta_{I_{i, k} \mid \tau_{i}}\left(\omega_{1}, \omega_{2}\right)$, let us show that

$$
\mathscr{L}_{i, k}^{(n)}=\left\{\begin{array}{l}
A^{2}+B^{2}=\left|\theta_{i, k}^{(n)}\left(l_{i}\right)\right|^{2}, \text { if } \theta_{i, k}^{(n)}\left(l_{i}\right)=\theta_{i, k}^{(n)}\left(l_{i}+1\right) \\
G^{2}\left(u_{i}-F\right)^{2}+H \quad \text { otherwise },
\end{array}\right.
$$

where

$$
\left\{\begin{array}{l}
A=\mathscr{R}\left\{\theta_{i, k}^{(n)}\left(l_{i}\right)\right\}, \quad B=\mathscr{G}\left\{\theta_{i, k}^{(n)}\left(l_{i}\right)\right\}, \\
C=\mathscr{R}\left\{\theta_{i, k}^{(n)}\left(l_{i}\right)\right\}, \quad J=\mathscr{G}\left\{\theta_{i, k}^{(n)}\left(l_{i}\right)\right\},
\end{array}\right.
$$

and in the case of $\theta_{i, k}^{(n)}\left(l_{i}\right) \neq \theta_{i, k}^{(n)}\left(l_{i}+1\right)$, the parameters are defined as

$$
\left\{\begin{array}{l}
E=\sqrt{(C-A)^{2}+(J-B)^{2}}=\left|\theta_{i, k}^{(n)}\left(l_{i}+1\right)-\theta_{i, k}^{(n)}\left(l_{i}\right)\right| ; \\
F=\frac{A^{2}+B^{2}-A C-B J}{(C-A)^{2}+(D-B)^{2}} ; \\
H=\frac{(A J-B C)^{2}}{(C-A)^{2}+(J-B)^{2}} .
\end{array}\right.
$$

In this case

$$
\begin{aligned}
J_{i, k}^{(n)}=\mid & {[(C-A)+j(J-D)] u_{i}+A+\left.j B\right|^{2}=\left[(C-A) u_{i}\right.} \\
+A]^{2}+\left[(J-B) u_{i}+B\right]^{2}=\left[(C-A)^{2}+(J-B)^{2}\right] u_{i}^{2} & \\
& +2[(C-A) A+(J-B) B] u_{i}+A^{2}+B^{2} \\
= & \left\{\begin{array}{l}
A^{2}+B^{2},\left|\theta_{i, k}^{(n)}\left(l_{i}+1\right)=\theta_{i, k}^{(n)}\left(l_{i}\right)\right| \\
E^{2}\left(u_{i}-F\right)^{2}+H, \text { otherwise }
\end{array}\right.
\end{aligned}
$$

Consider the next statement. Let

$$
\begin{gathered}
\varphi_{1}^{(n)}\left(l_{i}, \omega_{1}, \omega_{2}\right) \\
\cong \int_{0}^{1} \exp \left\{-\left(\omega_{1}^{2}+\omega_{2}^{2}\right) P_{i} T_{c}^{2}\left[E^{2}\left(u_{i}-F\right)^{2}+H\right] d u_{i} .\right.
\end{gathered}
$$

Then

$$
=\frac{\varphi_{1}^{(n)}\left(l_{i}, \omega_{1}, \omega_{2}\right)}{T_{c} E \sqrt{2 \pi} \mathscr{M}_{1}(i)} \exp \left\{-\left(\omega_{1}^{2}+\omega_{2}^{2}\right) P_{i} T_{c}^{2} H\right\},
$$

where

$$
\begin{aligned}
\mathcal{M}_{1}(i) & =Q\left[T_{c} E(F-1) \sqrt{2 P_{i}\left(\omega_{1}^{2}+\omega_{2}^{2}\right)}\right] \\
& \left.-Q\left[T_{c} E F\right) \sqrt{2 P_{i}\left(\omega_{1}^{2}+\omega_{2}^{2}\right)}\right] .
\end{aligned}
$$

Based on definition [32] we obtain

$$
\begin{aligned}
& \varphi_{1}^{(n)}\left(l_{i}, \omega_{1}, \omega_{2}\right)=\exp \left\{-\left(\omega_{1}^{2}+\omega_{2}^{2}\right) P_{i} T_{c}^{2} H\right\} \\
& \times \int_{0}^{1} \exp \left\{-\left(\omega_{1}^{2}+\omega_{2}^{2}\right) P_{i} T_{c}^{2}\left[E^{2}\left(u_{i}-F\right)^{2}\right]\right\} d u_{i} \\
= & \frac{1}{T_{c} E \sqrt{2 P_{i}\left(\left(\omega_{1}^{2}+\omega_{2}^{2}\right)\right.}} \exp \left\{-\left(\omega_{1}^{2}+\omega_{2}^{2}\right) P_{i} T_{c}^{2} H\right\}
\end{aligned}
$$

$$
\times \int_{T_{c} E(F-1) \sqrt{2 P_{i}\left(\omega_{1}^{2}+\omega_{2}^{2}\right.}}^{\int_{c} E F \sqrt{2 P_{i}\left(\omega_{1}^{2}+\omega_{2}^{2}\right.}} \exp \left(-0.5 t^{2}\right) d t .
$$

Thus, (70) is obtained. Now, the characteristic function of $I_{i, k}$ can be presented in the following form:

$$
\Theta_{I_{i, k}}\left(\omega_{1}, \omega_{2}\right)=\frac{1}{4 T} \sum_{n=0}^{3} \sum_{l_{i}=0}^{N-1} \varphi^{(n)}\left(l_{i}, \omega_{1}, \omega_{2}\right),
$$

where

$$
=\left\{\begin{array}{c}
\varphi^{(n)}\left(l_{i}, \omega_{1}, \omega_{2}\right) \\
\exp \left\{-\left(\omega_{1}^{2}+\omega_{2}^{2}\right) P_{i} T_{c}^{2}\left|\theta_{i, k}^{(n)}\left(l_{i}\right)\right|^{2},\right. \\
\left|\theta_{i, k}^{(n)}\left(l_{i}+1\right)=\theta_{i, k}^{(n)}\left(l_{i}\right)\right| \\
\varphi_{1}^{(n)}\left(l_{i}, \omega_{1}, \omega_{2}\right), \quad \text { otherwise }
\end{array}\right.
$$

with $\varphi_{1}^{(n)}\left(l_{i}, \omega_{1}, \omega_{2}\right)$ is given by (70). Note that the above characteristic function is given as an explicit closed-form expression involving only the exponential function and Q-functions. It can be readily programmed for direct evaluation. Moreover, it is easy to show that

$$
\varphi_{1}^{(n)}\left(l_{i}, \omega_{1}, \omega_{2}\right) \rightarrow \exp \left\{-\left(\omega_{1}^{2}+\omega_{2}^{2}\right) P_{i} T_{c}^{2}\left|\theta_{i, k}^{(n)}\left(l_{i}\right)\right|^{2}\right.
$$

as

$$
\theta_{i, k}^{(n)}\left(l_{i}\right)-\theta_{i, k}^{(n)}\left(l_{i}+1\right) \mid \rightarrow 0 .
$$

Since the complex random variables $I_{i, k}, 1 \leq i \leq K$, $i \neq k$ from different interferers are independent, the characteristic function for the total multiple-access interference term $I_{i}$ is given by

$$
\Theta_{I_{i, k}}\left(\omega_{1}, \omega_{2}\right)=\prod_{i=1, i \neq k}^{K} \Theta_{I_{i}}\left(\omega_{1}, \omega_{2}\right) .
$$

Let $\xi_{i}=I_{i}+\varsigma_{i}$, where $\varsigma_{i}$ is the complex zero-mean Gaussian random variable in the statistical sense representing the background noise [14]. Since the other user interference and background noise are independent, the characteristic function of $\xi_{i}$ takes the following form:

$$
\Theta_{\xi_{i}}\left(\omega_{1}, \omega_{2}\right)=\Theta_{I_{i}}\left(\omega_{1}, \omega_{2}\right) \Theta_{\varsigma_{i}}\left(\omega_{1}, \omega_{2}\right),
$$

where $\Theta_{\zeta_{i}}\left(\omega_{1}, \omega_{2}\right)$ is the characteristic function of the background noise $\varsigma_{i}$. Then the marginal characteristic functions of $\xi_{i}$ are given by

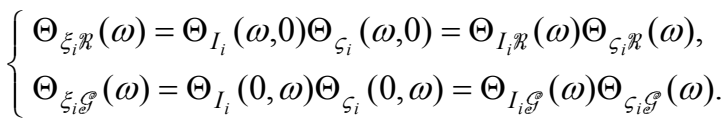

It follows from (73)-(79) that the marginal characteristic functions of $\xi_{i}=I_{i}+\varsigma_{i}$ satisfy the following equality $\Theta_{\xi_{i} R}(\omega)=\Theta_{\xi_{i} g}(\omega)$. Therefore, by symmetry, the conditional $B E R$ for the target user can be given

$$
\begin{aligned}
\overline{B E R}_{A_{i}}= & 0.5\left(\overline{B E R}_{i \mathscr{R} \mid A_{i}}+\overline{B E R}_{i \mathscr{G} \mid A_{i}}\right)=\overline{B E R}_{i \mathscr{R} \mid A_{i}} \\
& =B E R\left\{\mathscr{R}\left(\xi_{i}\right)<-\sqrt{P_{i}} A_{i} T\right\}
\end{aligned}
$$




$$
\begin{gathered}
=\frac{1}{2}-\frac{1}{\pi} \int_{0}^{\infty} \frac{\sin \left(\sqrt{P_{i}} A_{i} T \omega\right)}{\omega} \Theta_{\xi_{i} R}(\omega) d \omega \\
=Q\left(\frac{\sqrt{P_{i}} A_{i} T}{\sigma_{\zeta_{i} R}}\right)+\frac{1}{\pi} \int_{0}^{\infty} \frac{\sin \left(\sqrt{P_{i}} A_{i} T \omega\right)}{\omega}\left[1-\Theta_{I_{i} R}(\omega)\right] \\
\times \Theta_{\zeta_{i} R}(\omega) d \omega .
\end{gathered}
$$

Using the integral identity

$$
\int_{0}^{\infty} \sin (k x) x \exp \left(-0.5 x^{2}\right) d x=\sqrt{0.5 \pi} k \exp \left(-0.5 k^{2}\right)
$$

and averaging over $A_{i}$, we obtain the average $B E R$ for a Rayleigh faded user given by

$$
\begin{gathered}
B E R=\frac{1}{2}\left\{1-\left(\sqrt{1+\frac{N_{0}^{2}}{16 E_{i}^{2}}}\right)^{-1}\right\} \\
+\sqrt{\frac{P_{i}}{2 \pi}} T \int_{0}^{\infty} \exp \left(-0.5 P_{i} T^{2} \omega^{2}\right)\left[1-\Theta_{I_{i} R}(\omega)\right] \Theta_{\zeta_{i} R}(\omega) d \omega .
\end{gathered}
$$

Consider the DS-SSMA quaternary asynchronous system with complex sequences and complex receivers over Rayleigh fading channels shown in Fig. 1. If the chip waveform is a rectangular pulse, then the average $B E R$ of Rayleigh-faded user can be given by (82). Note that the above-mentioned $B E R$ in (82) is expressed as the $Q$-functions and single integral. Numerical integration techniques similar to [33] that uses Simpson's rule and series expansion method can be applied to obtain $B E R$ with high accuracy.

\subsubsection{Gaussian Approximation}

The high accuracy $B E R$ for asynchronous system under consideration can be obtained from previous discussion. However, it would generally require excessive computations. Hence, here we shall compute the average $B E R$ for asynchronous complex DSSSMA system in flat Rayleigh fading by modeling multiple-access interference in (34) as a complex Gaussian process. Obviously, the mean of the multiple-access interference reduce to zero and the variance is given by

$$
\begin{gathered}
\sigma_{I_{k} R}^{2}=\sigma_{I_{k} \mathscr{E}}^{2}=\sum_{i=1, i \neq k}^{K} 2 P_{i} E\left[\left|R_{i, k}\left(\tau_{i}\right)\right|^{2}+\left|\hat{R}_{i, k}\left(\tau_{i}\right)\right|^{2}\right] \\
=\sum_{i=1, i \neq k}^{K} \frac{2 P_{i}}{T} \int_{0}^{T}\left[\left|R_{i, k}\left(\tau_{i}\right)\right|^{2}+\left|\hat{R}_{i, k}\left(\tau_{i}\right)\right|^{2}\right] d \tau \\
=\sum_{i=1, i \neq k}^{K} \sum_{k=0}^{N-1} \frac{2 P_{i}}{T} \int_{l T_{c}}^{(l+1) T_{c}}\left[\left|R_{i, k}\left(\tau_{i}\right)\right|^{2}+\left|\hat{R}_{i, k}\left(\tau_{i}\right)\right|^{2}\right] d \tau,
\end{gathered}
$$

where the mathematical expectation has been computed with respect to mutually independent complex random variables $\left.\left\{A_{i}, \varphi_{i}, b_{-1}^{(i)}, b_{0}^{(i)}\right\}\right|_{i=1} ^{K}$ and the random variables given by (54) being zero-mean complex Gaussian with the unit variance. It is trivial to show that

$$
E\left[\mathscr{R}\left(I_{k}\right) \mathscr{P}\left(I_{k}\right)\right]=E\left[\mathscr{R}\left(I_{k}\right)\right] E\left[\mathscr{\mathscr { S }}\left(I_{k}\right)\right]=0
$$

with that, the real and imaginary parts of random variable $I_{k}$ are uncorrelated and orthogonal. Therefore, if $I_{k}$ is assumed to be complex Gaussian random variable, then it is independent. In the case of a rectangular wave shape function $\psi(t)=1,0 \leq t \leq T_{c}$, then $R_{\psi}(s)=s$ and $\hat{R}_{\psi}(s)=T_{c}-s$. Hence, following the treatment in [4] and substituting (37) in (83), we obtain

$$
\sigma_{I_{k} \mathscr{R}}^{2}=\sigma_{I_{k} \mathscr{E}}^{2}=\sum_{i=1, i \neq k}^{K} \frac{2 P_{i} T^{2}}{3 N^{3}} r_{i, k}
$$

where

$$
\begin{gathered}
r_{i, k}=\sum_{l=0}^{N-1}\left[C_{i, k}(l-N) C_{i, k}^{*}(l-N)+C_{i, k}(l+1-N)\right. \\
\times C_{i, k}^{*}(l+1-N)+C_{i, k}(l) C_{i, k}^{*}(l)+C_{i, k}(l+1) C_{i, k}^{*}(l+1) \\
\mathscr{R}\left\{C_{i, k}(l-N) C_{i, k}^{*}(l+1-N)\right\}+\mathscr{R}\left\{C_{i, k}(l) C_{i, k}^{*}(l+1)\right\} \\
=2 \mu_{i, k}(0)+\mathscr{R}\left\{\mu_{i, k}(1)\right\}
\end{gathered}
$$

with

$$
\mu_{i, k}(n)=\sum_{l=1-N}^{N-1} C_{i, k}(l) C_{i, k}^{*}(l+n) .
$$

Similar to analysis for the synchronous case, given $A_{k}$, the average conditional $B E R \mathrm{~s}$ for the real and imaginary parts of the $i$-th user in the asynchronous case are

$$
B E R_{k|\mathcal{R}| A_{k}}=B E R_{k \mathscr{g} \mid A_{k}}=Q\left\{\frac{\sqrt{P_{k}} A_{k} T}{\sqrt{\sum_{i=1, i \neq k}^{K} \frac{2 P_{i} T^{2}}{3 N^{3}} r_{i, k}+\frac{N_{0}^{2}}{16}}}\right\} .
$$

Averaging over $A_{k}$ with respect to the Rayleigh distribution in (18), the average asynchronous $B E R$ for a Rayleigh-faded user takes the following form:

$$
\overline{B E R}=0.5\left(\overline{B E R}_{i \Re}+\overline{B E R}_{i \mathscr{G}}\right)=0.5\left(1-\Delta_{a}\right)
$$

where

$$
\Delta_{a}=\frac{1}{\sqrt{1+\sum_{i=1, i \neq k}^{K} \frac{2 P_{i}}{3 N^{3} P_{k}} r_{i, k}+\frac{N_{0}^{2}}{16 E_{k}^{2}}}} .
$$

Based on the Gaussian approximation of the multiple-access interference, the average $B E R$ evaluation is approximately obtained in (89) for quaternary asynchronous DS-SSMA system with complex signature sequences, as well as complex transmitters and rece- 
ivers in Rayleigh fading as depicted in Fig.1.

From [25]

$$
\sum_{l=1-N}^{N-1} C_{i, k}(l) C_{i, k}^{*}(l+n)=\sum_{l=1-N}^{N-1} C_{i}(l) C_{k, k}^{*}(l+n) .
$$

Therefore

$$
r_{i, k}=2 N^{2}+4 \sum_{l=1}^{N-1} C_{i}(l) C_{k, k}^{*}(l)+\mathscr{R}\left\{\sum_{l=1-N}^{N-1} C_{i}(l) C_{k}^{*}(l+1)\right\}
$$

This shows that the $B E R$ performance for asynchronous system over Rayleigh fading channel can be obtained from aperiodic autocorrelation functions.

\section{Numerical Results}

In this section, we report the numerical evaluation of the system performance for Gold sequences, ne-aroptimum four phase sequences of family of [34] and UCHT sequences. As was discussed previously, there are 64 sets of UCHT matrices, and each set can generate $2^{n}$ complex sequences with the period duration of $N=2^{n}$. It has been investigated in [10] that not all the UCHT sequences generated from one UCHT matrix can be simultaneously used as the spreading sequences, as this will lead to large periodic and aperiodic cross correlation values.

In order to reduce the multiple-access interference effectively, the subset of the complex sequences with smaller sum of aperiodic cross correlation values is selected for simulation, but a consideration of trade-off between the autocorrelation and cross correlation properties for spreading sequences suggests that the subset with the smallest sum must be avoided. High-speed UCHT sequences are also recommended to be more suitable for the asynchronous DSSSMA communication systems than non-high speed UCHT sequences [9].

Hence, in this section, we will choose $\mu_{1}=j, \mu_{2}$ $=1, \mu_{3}=j$ and $N=2^{6}=64$ as one set of non-highspeed UCHT sequences which have relatively good autocorrelation and cross correlation among the UCHT sequences [10]. On the other hand, the Gold sequences are produced by a preferred pair of $m$-sequences $u$ and $v$, where $u$ is obtained by using primitive polynomials

$$
f_{1}=x^{6}+x+1
$$

and $v$ is obtained by using

$$
f_{2}=x^{6}+x^{5}+x^{2}+x+1 \text {. }
$$

Thus, the period of the Gold sequences is $N=63$, and it has 65 different sequences belonging to the set $\mathscr{P}(u, v)=\left\{u, v, u \oplus v, u \oplus \mathscr{T} v, u \oplus \mathscr{T}^{2} v, \ldots, u \oplus \mathscr{T}^{N-1} v\right\}$ (95) where $\mathscr{T}$ is the shift operator. The 4-phase family of sequences are produced by using the primitive polynomials

$$
f_{3}=x^{6}+2 x^{3}+3 x+1
$$

So, the period of 4-phase sequences is $N=63$ with 65 different sequences. In the simulations, equal transmission power, for simplicity, is assumed for each user and

$$
P_{1}=P_{2}=\cdots=P_{K}=1 .
$$

The BER performance of quaternary synchronous DS-SSMA system over Rayleigh fading channels is shown in Figs. 3 and 4, where the active users are $K$ $=8, K=16$ and $K=30$, respectively.

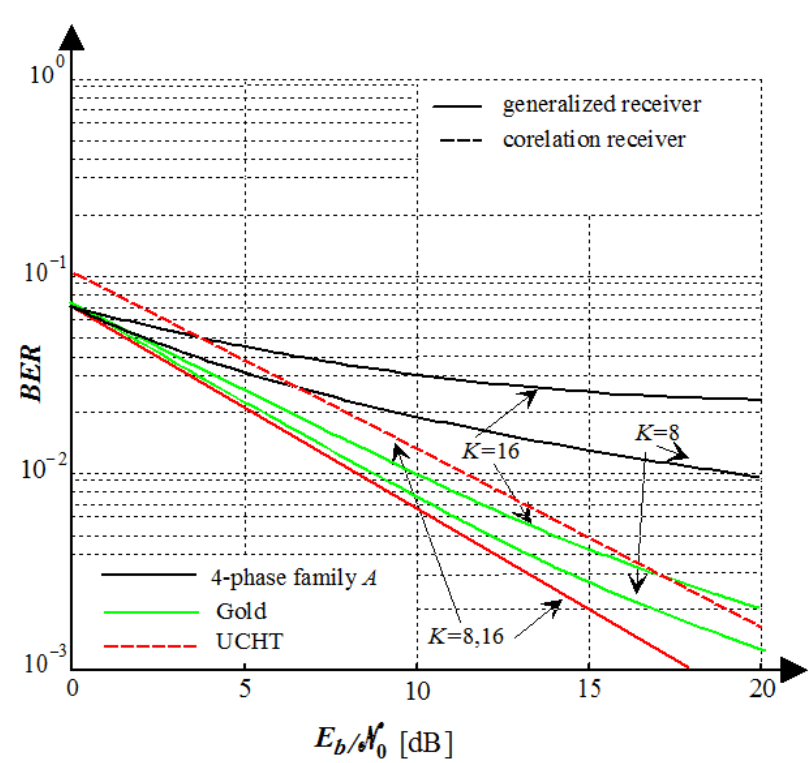

Fig. 3. BER performance for synchronous DS-SSMA with active users $K=8,16$.

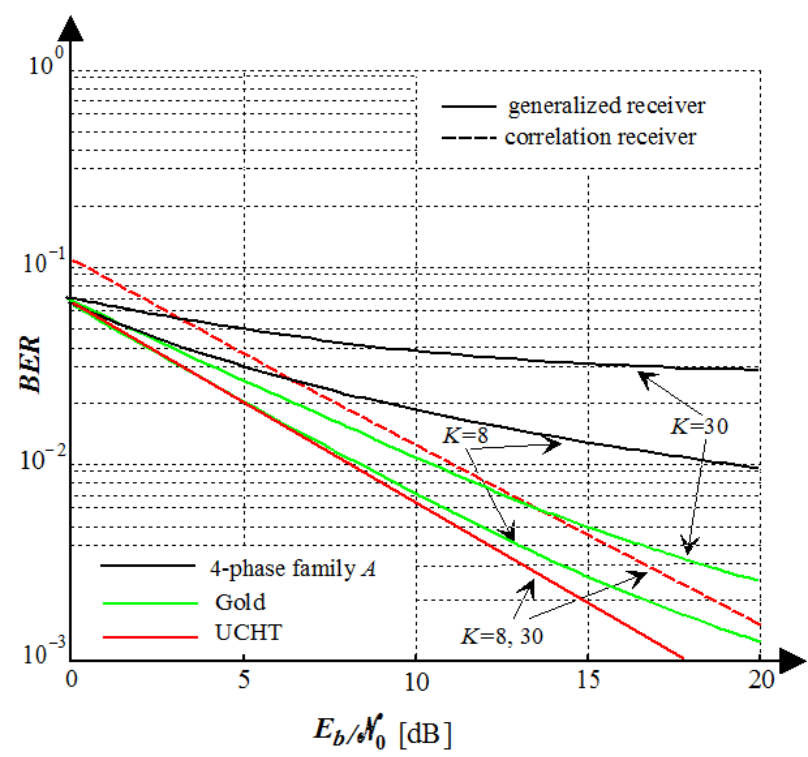

Fig. 4. BER performance for synchronous DS-SSMA with active users $K=8,30$. 
It can be seen that the performance of the UCHT sequences is the best among the three sequences, and the Gold sequences also perform better than 4phase family $A$ sequences at the synchronous conditions. The reason is that UCHT sequences are orthogonal, i.e., $C_{i, k}(0)=0, i \neq k$. As to the Gold sequences, $C_{i, k}(0)-1$, if $i \neq k$ and the $v$ in the set of Gold sequences $\mathscr{P}(u, v)$ is not chosen as spreading sequence. For The 4-phase family of sequences, $C_{i, k}(0)=0, i \neq k$, could be $-1,7,-9,-1 \pm 8 j$ and each occurs many times. Hence, in view of (42), the BER performance in Figs. 3 and 4 are reasonable. Furthermore, for UCHT sequences, the curves of $B E R \mathrm{~s}$ are the same for all different number of users, such as $K=8,16,30$. However, for the Gold sequences and 4-phase family of sequences, the curves of $B E R \mathrm{~s}$ are different for $K=$ $8,16,30$.

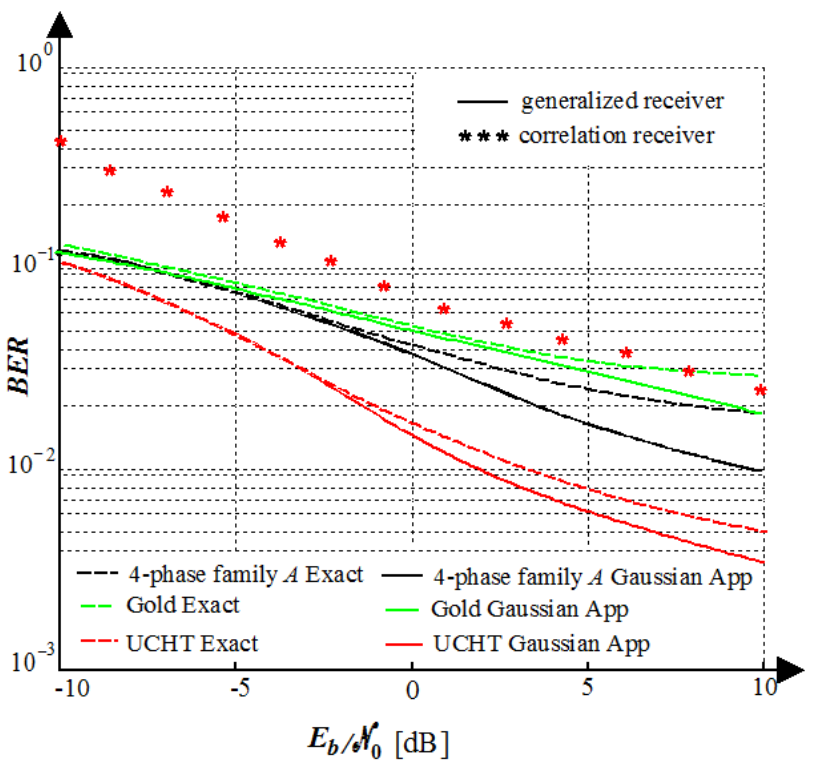

Fig. 5. BER performance for asynchronous DS-SSMA with active users $K=8$. Correlation receiver is represented for UCHT and Gaussian approximation.

Finally, Figs. 5-7 show the accurate $B E R$ computed using the characteristic function approach as well as the $B E R$ obtained by the Gaussian approximation method described in the previous section for quaternary asynchronous DS-SSMA systems with the Rayleigh fading channels, where the active users are $K=8,16,30$, respectively. In the asynchronous case, the cross correlation of the Gold sequences, $C_{i, k}(l), i \neq k,|l|, 63$, could be $-1,-17,15$ and each value occurs many times. The distributions of aperiodic cross correlation values are different for the Gold and 4-phase family of sequences, but they are similar. Thus, the Gold and 4-phase family of sequences belong to pseudo-random spreading sequences and have similar correlation properties.

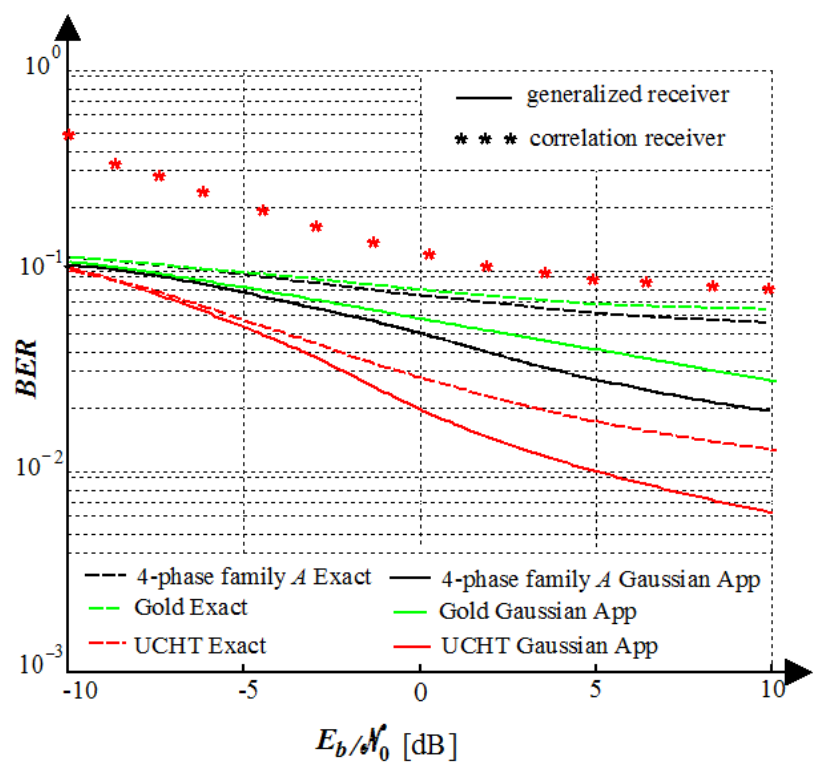

Fig. 6. BER performance for asynchronous DS-SSMA with active users $K=16$.

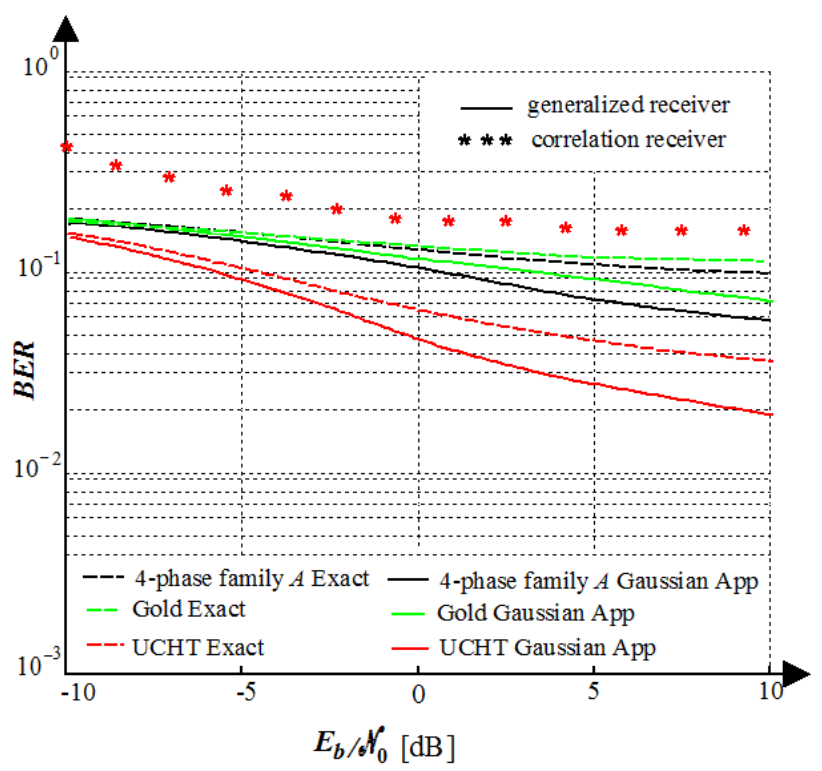

Fig. 7. BER performance for asynchronous DS-SSMA with active users $K=30$.

It has also been shown that the UCHT complex sequences offer the better cross correlation properties compared with the Gold sequences [10]. In view of (82) and (89), the BER performance is mainly determined by the cross correlation properties of the signature sequences. Figures 5-7 have verified this. That is to say, the asynchronous system under consideration with UCHT sequences outperforms that with the Gold and 4-phase family of sequences, and the BER performance using the Gold sequences is also close to that using the 4-phase family of sequences.

We also derive from our numerical results prese- 
nted in Figs. 5-7 that the BER estimates based on the complex Gaussian approximation consistently overestimate the accurate $B E R$ computed via the characteristic function method for the asynchronous quarternary DS-SSMA system in the Rayleigh fading channels with complex processing at the transmitter and receiver. Comparative analysis between the asynchronous DS-SSMA systems constructed on the basis of the generalized receiver and correlation one is represented in Figs. 5-7. We can see superiority in the $B E R$ performance under employment of the generalized receiver in DS-SSMA communication systems with active users in comparison with implementation of the correlation one in these systems.

\section{Conclusions}

This paper investigates the performance of quaternary DS-SSMA communications, which are constructed on the basis of the generalized approach to signal processing in noise, with complex signature sequences, as well as complex transmitters and receivers over the Rayleigh fading channels. The average $B E R$ is first derived for the quaternary synchronous systems. Due to the orthogonal property of UCHT sequences, the probability of bit errors of the synchronous DS-SSMA systems with UCHT sequences is lower in comparison with the synchronous systems with other non-orthogonal sequences.

The average $B E R$ is also evaluated for the quarternary asynchronous DS-SSMA systems with complex transmitters and generalized receivers based on the characteristic function approach, and the approximate result is also given based on the Gaussian approximation method of multiple-access interference. Numerical results are presented to illustrate performance comparisons among systems employing the UCHT sequences, 4-phase family of sequences and Gold sequences. The simulation results show that the UCHT complex sequences can yield the better performance than other two sequences. Comparative analysis of the asynchronous DS-SSMA systems employing the generalized receiver demonstrates a superiority in of $B E R$ performance over asynchronous DS-SSMA systems implementing the correlation receiver.

\section{References:}

[1] Lee J.S. and Miller L.E., CDMA Systems Engineering Handbook. Artech House, Boston, MA., 1998.

[2] Frank, R.L. and Zadoff, S.A., "Phase shift pulse codes with good periodic correlation properties". IEEE Transactions on Information Theory.

1962. Vol. 8. No. 5, pp. 381-382.
[3] Torrier, D., Principles of Spread-Spectrum Communication Systems. Springer, Switzerland. 2018.

[4] Pursley, M.B., "Performance evaluation for phase-code spread-spectrum multiple-access communication-Part I: System analysis," IEEE Transactions on Communications. 1977. Vol. 25, No. 8, pp. 795-799.

[5] Viterbi, A.J., CDMA: Principles of Spread Spectrum Communication. Reading, MA: AddisonWesley, USA. 1995.

[6] Chu, D.C., "Polyphase codes with good periodic correlation properties," IEEE Transactions on Information Theory. 1972. Vol. 18. No. 4, pp. 531-532.

[7] Sarwate, D.V., "Bounds n cross-correlation and autocorrelation of sequences," IEEE Transactions on Information Theory. 1979. Vol. 25. No. 6, pp. 720-724.

[8] Lam, A.W. and Ozluturk, F.M., "Performance bounds for DS-SSMA communications with complex signature sequences," IEEE Transactions on Communications. 1992. Vol. 40. No. 10, pp. 1607-1614.

[9] Ozluturk,F.M., Tantaratana, S., Lam, A.W.,"Performances for DS-SSMA communications with MPSK signaling and complex signature sequences", IEEE Transactions on Communications. 1995. Vol. 43. No. 2/3/4, pp. 1127-1133.

[10] Rahardja, S., Ser, W., Lin, Z., "UCHT-based complex sequences for synchronous CDMA system." IEEE Transactions on Communications. 2003. Vol. 51. No. 4, pp. 618-626.

[11] Rahardja, S., Falkowski, B.J., "Family of unified complex Hadamard transforms," IEEE Transactions on Circuits and Systems II. 1999, Vol.46, no. 8, pp. 1094-1100.

[12] LehnertJ.S., Pursley, M.B., "Error probabilities for binary direct-sequences spread-spectrum communications with random signature sequences." IEEE Transactions on Communications. 1989. Vol. 37. no. 8, pp. 851-858.

[13] Tuzlukov, V.P., "A new approach to signal detection theory," Digital Signal Processing, 1998, Vol. 8, No. 3, pp. 166-184.

[14] Tuzlukov, V.P., Signal Detection Theory, New York: Springer-Verlag, 2001.

[15] Tuzlukov, V.P., Signal Processing Noise, Boca Raton, London, New York, Washington D.C.: CRC Press, Taylor \& Francis Group, 2002.

[16] Tuzlukov, V.P., "DS-CDMA downlink systems with fading channel employing the generalized”, Digital Signal Processing, 2011. Vol. 21, No. 6, pp. 725-733.

[17] Tuzlukov, V.P., "Signal processing by generali- 
zed detector in DS-CDMA wireless communication systems with frequency-selective channels", Circuits, Systems, and Signal Processing, published on-line on February 2, 2011, doi:10. 1007/s00034-011-9273-1; 2011,Vol.30, No.6, pp. 1197-1230.

[18] Shbat, M., Tuzlukov, V.P. "Primary signal detection algorithms for spectrum sensing at low SNR over fading channels in cognitive radio." Digital Signal Processing (2019). https://doi. org/10.1016/j.dsp. 2019.07.16. Digital Signal Processing. 2019. Vol. 93. No. 5, pp. 187- 207.

[19] Borth, D.E., Pursley, M.B., "Analysis of directsequence spread spectrum multiple-access communication over Rician fading channels", IEEE Transactions on Communications. 1979. Vol. 27. No. 10, pp. 1566-1577.

[20] Gardner, C.S., Orr, J.A., "Fading effects on the performance of a spread spectrum multiple-access communication system", IEEE Transactions on Communications. 1979. Vol. 27. No.1, pp. 143-149.

[21] Cho, J.H., Jeomg, Y.K., Lehnert, J.S., “Average bit-error rate performance of band-limited DS-SSMA communications." IEEE Transactions on Communications. 2002. Vol. 50. No. 7 , pp. 1150-1159.

[22] Trabelsi, C., Yongacoglu, A., "Bit-error-rate performance for asynchronous DS-CDMA over multipath fading channels", IEE Proceedings Communications, 1980. Vol.142, No.5, pp.307314.

[23] Minić, S., Krstić, D., Bandjur, D., Milenković, V., Suad Suljović, S., Stefanović, M., "Level crossing rate of macrodiversity in the presence of gamma long-term fading, $\kappa-\mu$ short term fading and Rayleigh short-term fading", WSEAS Transactions on Communications, 2017.Vol.16, Art. \#1, pp. 1-7.

[24] Wallis, W.D., Street, A.P., Wallis, J.S. Combinatorics: Room Squares, Sum-Free Sets, Hadamard Matrices. New York: Springer-Verlag, 1972.

[25] Sarwate, D.V., Pursley, M.B., "Cross-correlation properties of pseudorandom and related sequences",Proceedings IEEE, 1980. Vol.68, No.5 pp.593-619.

[26] Maximov, M., "Joint correlation of fluctuative noise at outputs of frequency filters," Radio Engineering. 1956, No. 9, pp. 28-38.

[27] Chernyak, Y., "Joint correlation of noise voltage at outputs of amplifiers with nonoverlapping responses," Radio Physics and Electronics, 1960, No. 4, pp. 551-561.

[28] Shbat, M., Tuzlukov, V.P., "Evaluation of dete- ction performance under employment of the generalized detector in radar sensor systems," Radioengineering, 2014, Vol. 23, No. 1, pp. 50-65.

[29] Shbat, M., Tuzlukov, V.P., "Definition of adaptive detection threshold under employment of the generalized detector in radar sensor systems," IET Signal Processing, 2014, Vol. 8, Issue 6, pp. 622-632.

[30] Shbat, M., Tuzlukov,V.P., "SNR wall effect alleviation by generalized detector employment in cognitive radio networks," Sensors, 2015, 15 (7), pp.16105-16135; doi:10.3390/ s150716105.

[31] Macdonald, T.G. and Pursley, M.B., "The performance of direct-sequence spread-spectrum with complex processing and quaternary data modulation." IEEE Journal of Selected Areas of Communications. 2000, Vol. 18, No. 8, pp. 1408-1417.

[32] Irshid, M.I., Salous, I.S., "Bit error probability for coherent $M$-ary PSK systems", IEEE Transactions on Communications. 1991. Vol. 39. No. 3, pp. 349-352.

[33] Geraniotis, E.A., Pursley, M.B., "Error probability for direct-sequence spread-spectrum multiple access communications-Part II: Approximations", IEEE Transactions on Communications. 1991. Vol. 39. No. 3, pp. 349-352.

[34] Boztas, S., Hammons, R., Kumar, P.V., “4-phase sequences with near optimum correlation properties", IEEE Transactions on Communications. 1991. Vol. 39. No. 3, pp. 349-352. 